\title{
On the Urgent Need for Successful Randomized Controlled Trials in Neurosciences
}

Scientifically valid studies provide the best estimates of the truth, which is why we trust their results. Valid studies of therapies rigourously comparing different interventions ensure that biases and confounders (factors which can inflate or deflate the effects of an intervention) are equally distributed between the groups under comparison ${ }^{1}$. In the ideal study, patients under comparison are identical in every respect except for the intervention of interest. The method to achieve this level of scientific validity was introduced only 60 years ago, when the first randomized controlled trial (RCT) was performed. Adoption by regulatory agencies of this methodology as the gold standard has forever changed the face of clinical research ${ }^{2}$. In RCTs, each study participant has the same random or nearrandom chance of being allocated to the experimental or to the control group as the next participant; classically this is a 50:50 chance when randomization occurs in a 1:1 ratio, but other randomization ratios and methods are used depending upon the clinical question being tested. Because not knowing which treatment the next patient will receive is essential, allocation is concealed. In addition, outcomes are assessed in an identical manner in all patients, a process achieved by blinding study participants and research personnel to the treatment the patients are receiving, or by measuring outcomes in an independent, blinded manner. Inadequate concealment of randomization and inadequate blinding may overestimate treatment effect by up to $40 \%$ and $17 \%$, respectively ${ }^{3}$. The rigour of RCTs, central to evidence based therapy, is the gold standard to assess the benefit of interventions. The RCT is the now the corner stone of therapy testing. Time-honoured remedies that failed the scrutiny of RCTs have been abandoned ${ }^{4}$, and new therapies must prove their merit in the court of RCTs before they are approved.

Over 12,000 RCTs are now published yearly, and the numbers keep growing 5 . However, crucial RCTs in the neurosciences remain undone. Stroke has been a graveyard of drug development in the last two decades with so many negative trials that pharmaceutical business investment has evaporated. Device development in stroke has taken advantage of less stringent regulation and made advances, only to see regulation begin to catch up. Neurodegenerative conditions have some modestly effective symptomatic treatments but nothing that is going to reduce the prevalence of these conditions due to the silver tsunami as the first baby boomers hit age 65 this year. Prevalent neuromuscular disorders such as diabetic neuropathy desperately need preventive treatments. Common neurological disorders - epilepsy, migraine, carpal tunnel syndrome, shingles, Parkinson's disease, Alzheimer's/mixed dementia - all require large scale trials to address common clinical treatment questions. So, why aren't there more successful RCTs in the neurosciences?
The success of RCTs is threatened at many levels, from design through execution to publication. At the design stage, critical thinking of whether the clinical question is best answered by an RCT needs to be undertaken early; it is wrong to believe that every question is a nail to be hit by the RCT hammer. Unnecessary complexity, overly stringent eligibility criteria, and unresponsive, meaningless or unreliable outcome measures, all decrease the probability of successful implementation or of detecting a significant treatment effect. We need simple, easily implementable designs, with clear but simple eligibility criteria and carefully chosen, simple, responsive and clinically relevant outcome measures. Trials resembling clinical practice are much more likely to be successful. Further education on the nuances and functions of trial design and randomization would facilitate trial conduct, and reduce the need for burdensome complexity.

Approval by institutional review boards (IRB) poses important challenges for RCTs. Some of these include the need for multiple IRB approvals and contracts for a multicentre study, a lack of understanding of the importance of secure randomization and of patient identifiers for trial conduct and follow-up, the requirement for unwieldy consent forms, the difficulty in obtaining approval for use of drugs, and the long delays in obtaining ethics approvals. There is an ethical balance between the principles of individual autonomy and social justice. The balance in Canada has swung toward regulation to protect the individual ${ }^{6}$. We are well aware of past abuses in human experimentation and no one questions the need for serious and substantial safeguards for patient protection, but many feel that changes in regulations are urgently needed.

Funding for RCTs is limited and difficult to obtain. In addition, the cost of trials is increasing for two main reasons. First, the need for increasingly large trials requires substantial increases in funding. Second, the number and complexity of regulations for the conduct of RCTs increases their cost.

A fundamental obstacle to RCTs is difficulty in recruiting sufficient numbers of patients to find small but clinically important treatment effects, and this has several explanations. There is a realization that treatment effects of interventions in general have become progressively smaller over time, akin to a steady decline of therapeutic power of interventions. In some cases, the effects of new drugs seem to be less than half that of similar, older drugs introduced two decades ago. The stark reality is that the majority of interventions produce modest effects, and that RCTs need to enrol thousands and even tens of thousands of patients to demonstrate an effect. The proliferation of RCTs does not follow a proportionate increase in the number of potential participants. Thus, increasing number of RCTs compete for the same pool of potential patients. Finally, 
engagement of clinicians in the trenches, those who recruit or refer patients for recruitment, represents an enormous challenge. Clinicians may be unengaged for a number of reasons. For example, the "scientific clinical culture" may be poorly developed and there may be a lack of equipoise (or uncertainty) about the merit of the trial. In the Socratic sense, the more you learn clinically, the greater your uncertainty becomes (or should become!). Nevertheless, physicians are trained to make decisions, to act, often quickly and decisively, and not necessarily to randomize. Physicians may lack information about the intervention or the RCT, or the RCT may be too complex and unwieldy for the busy clinician. Perhaps, most importantly, enrolling patients is time consuming, requires meticulous attention to detail and is simply not well remunerated. In a culture where time is money, RCTs take second place.

Publication of RCTs is perhaps the final hurdle. Even well conducted, adequately powered RCTs can yield negative results. However, journals have little interest in publishing negative trials, or those with negligible effect sizes. After a new paradigm is proposed, the peer-review process is biased toward positive results. Yet, the publication of negative trials is extremely important. There is ample evidence in the literature of initially positive treatment effects that upon replication not only become less positive, but actually become solidly negative. Evidence for useless or harmful interventions is as important as that for helpful and safe treatments 7,8 .

The well-conducted RCT is an artful mixture of scientific method, ethics, politics, grant-writing, fund-raising, management and communication. Researchers should encourage public debate about how best to strike the balance between regulation and cost, how to stimulate reform and simplification of RCTs while enhancing patient safety and autonomy, and how to improve the scientific validity and integrity of RCTs while making them more affordable.

\section{REFERENCES}

1. Sackett DL. Bias in analytic research. J Chronic Dis. 1979;32: 51-63.

2. MRC Streptomycin in Tuberculosis Trials Committee. Streptomycin treatment for pulmonary tuberculosis. Br Med J. 1948;ii:769-82.

3. Schulz KF, Chalmers I, Hayes RJ, Altman DG. Empirical evidence of bias: dimensions of methodological quality associated with estimates of treatment effects in controlled trials. JAMA. 1995; 273(5):408-12.

4. The EC/IC Bypass Study Group. Failure of extracranial-intracranial arterial bypass to reduce the risk of ischemic stroke. N Engl J Med. 1985;313:1191-200.

5. Sackett DL, Hoey J. Why randomized controlled trials fail but needn't: a new series is launched. CMAJ. 2000 May 2;162(9): 1301-2.

6. Hebert PC, Fergusson DA. Are we keeping research participants safe enough? CMAJ. 2010 July 13;182(10):E428.

7. Lehrer J. The truth wears off. Is there something wrong with the scientific method? The New Yorker. 2010 December 13.

8. Ioannidis JP. Contradicted and initially stronger effects in highly cited clinical research. JAMA. 2005 July 13;294(2):218-28
Samuel Wiebe, Michael D. Hill

University of Calgary

Calgary, Alberta, Canada 